\title{
Données sur le parasitisme protélien de Nectonema (Nématomorphe), chez les Crustacés
}

\author{
Par Lucie ARVY
}

Soupçonnée par Müller (1871) chez les Pagures de Desterro (Ile Santa Catharina ; proche de la côte atlantique du Brésil) et par BATE (1888) chez un Penaeidae, pris par le «Challenger» au large de la Sierra Leone, l'existence d'un stade protélien du Nectonema, chez divers Crustacés, a été établie avec certitude par Pérez (1927-34) et les chercheurs de Roscoff (Mouchet, 1931 ; Bennati-Mouchet, 1934 ; Nouvel, 1934 ; Feyel, 1936).

Les Pagures semblent bien être les hôtes d'élection du Nectonema; de tous les Crustacés reconnus porteurs de Nématomorphe (Tabl.) ce sont les Pagures qui sont le plus fréquemment parasités (Müller, 1871 ; Pérez, 1927 ; Mouchet, 1931 ; Feyel, 1936). Cependant malgré de nombreuses dissections je n'ai jamais trouvé de Nectonema dans les divers Pagures observables à Dinard.

Les premières crevettes porteuses de Nectonema sont probablement celles qui ont été observées par BATE (1888) et par WARD (1892). BATE a dessiné un Pénaéide porteur d'un Nématomorphe; l'hôte identifié comme Gennadas parvus serait en réalité Amalopaeneus elegans, suivant ORTMANN (1935), appellation très probablement exacte comme le fait remarquer STEPHENSEN (1923) puisque ce Crustacé fut récolté sur la côte africaine de l'Atlantique et que les Gennadas parvus ne sont connus que du Pacifique et de l'Océan Indien.

Quoi qu'il en soit sur ce point, Nouvel (1934-6), à Roscoff (Finistère), GALliEN (1949) et moi-même (1956-62) sur les côtes du département de l'Ille-et-Vilaine, avons retrouvé l'infestation des crevettes par le Nectonema.

L'ensemble des observations faites depuis plus de neuf décades permettent de retenir un certain nombre de données qui pourraient orienter fructueusement les recherches futures. Ces données ont trait à l'identité des hôtes et du parasite, aux caractéristiques du parasitisme et à ses conséquences.

\section{Identité et sexe des hôtes.}

La spécificité parasitaire des hôtes n'est pas étroite puisque le Nectonema peut être trouvé chez des Pagures (Eupagurus et Anapagurus), des Diogenes, des Portunus, des Macropodia, des Munida ainsi que chez diverses crevettes: Palaemon, Crangon et Leander. 
Les deux sexes semblent être attaqués sans préférence, le plus souvent ; cependant les Eupagurus mâles observés par Mouchet (1931) étaient parasités dans $50 \%$ des cas, alors que les femelles ne l'étaient que dans $10 \%$ des cas.

\section{Identité des parasites.}

L'identité du Nématomorphe parasite reste le plus souvent inconnue. Qu'il s'agisse d'un Nectonema est indiscutable, puisque dans tous les cas, le parasite est filiforme, cœlomique, plus ou moins blanc, opaque ou translucide (fig. 1), porteur de deux brosses longitudinales, l'une dorsale, l'autre ventrale, constituées par de fines digitations de la cuticule. Les essais d'élevage des Nectonema parasites, tenté par Nouvel (1934), dans deux cas, n'a pas abouti : dans les deux cas, les parasites sont morts avant la mue de la crevette-hôte.

Feyel (1936) a identifié les Nectonema vus chez Anapagurus Hyndmanni Thompson et chez Macropodia rostrata (L.) ì Nectonema agile, Verrill, 1879.

\section{Taille et sexe des parasites.}

Tous les cas de parasitisme observés se rapportent à des stades avancés du développement du Nectonema.

La taille du parasite est souvent impressionnante et remarquée dès les premières observations (MüLler 1871); 6 à $8 \mathrm{~cm}$ sont des longueurs moyennes; des longueurs de $20 \mathrm{~cm}$ ont été rapportées, le parasite (fig. 1 à 4) occupant à lui seul plus de place dans le cœlome que tous les viscères de l'hôte réunis (MoucheT, 1931).

Les observateurs n'ont pas toujours précisé le sexe des Nectonema: il semble d'après les quelques précisions que nous possédons, que les deux sexes soient également fréquents.

\section{Unicité et pluralité des Nectonema.}

Le plus souvent l'hôte ne porte qu'un Nectonema cependant une infestation double est fréquente chez les Pagures (Mouchet, 1931) ; Pérez (1934) l'a notée 4 fois chez Macropodia, 5 fois chez Anapagurus et FeYel (1936) l'a vue 4 fois chez Anapagurus; une triple infestation a été observée par Mouchet (1931) et un Munida tenuimana hébergeait 6 Nectonema totalisant une longueur d'un mètre (BRINKMANN, 1930). Les parasites sont du même sexe ou de sexes différents dans ce dernier cas, les mâles sont toujours plus petits et plus translucides que les femelles.

\section{Conséquence du parasitisme.}

PÉrez (1927) et Nouvel (1934) sont les seuls à avoir estimé que les Crustacés parasités sont affectés, ayant en particulier des ovaires atrophiés dépourvus de gros ovocytes. BRINKMANN (1930), Mouchet (1931) n'ont pu déceler aucune anomalie morphologique ou fonctionnelle chez les Crustacés porteurs de Nectonema; les pléopodes et les gonades en particulier, ont leur type habituel ; la cicatrisation, la régénération, la crois- 

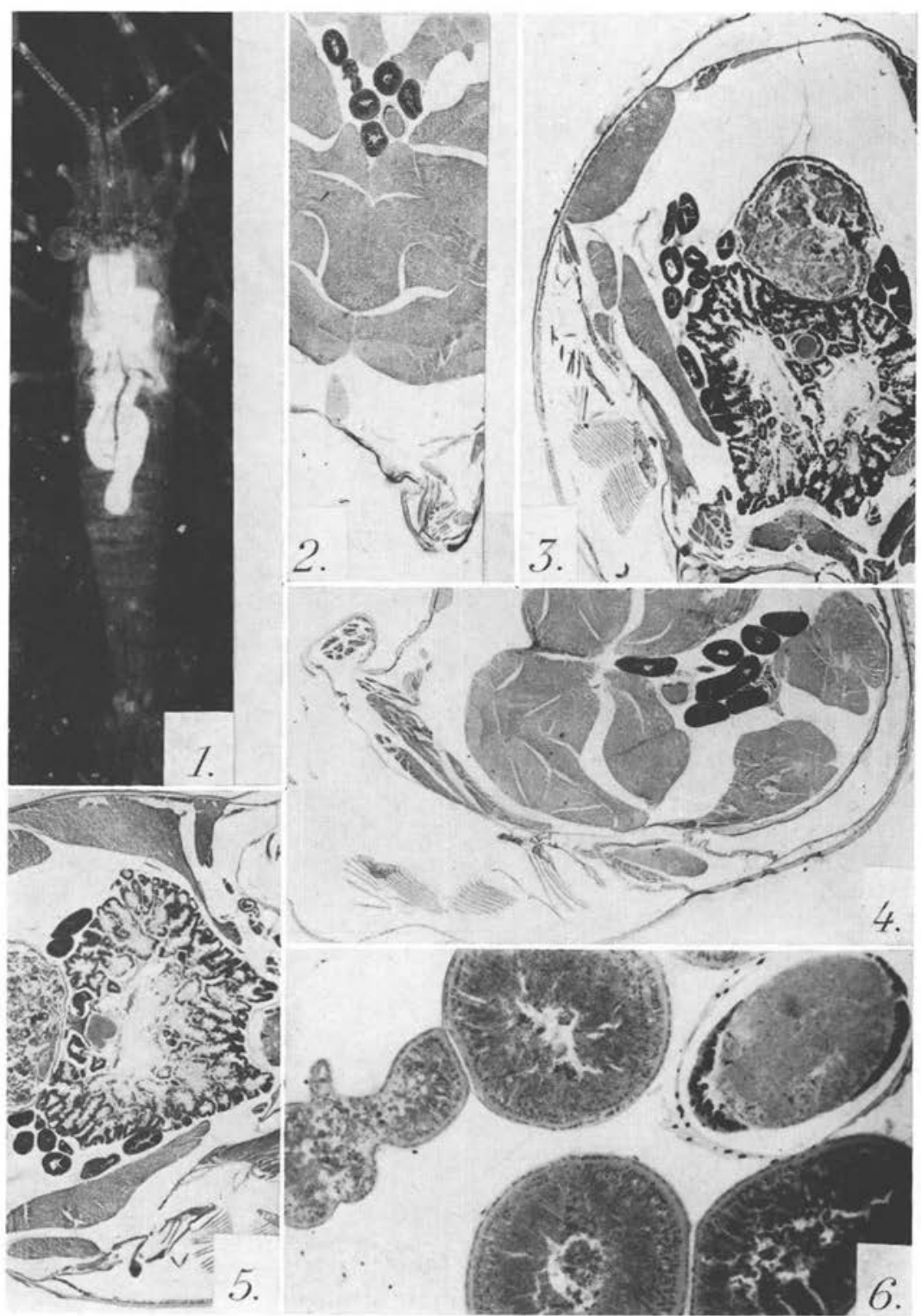

FIG. 1. - Nectonema parasite de Leander squilla L, photographié in vivo (Photographie du $\mathrm{D}^{\mathrm{r}}$ Rob Lami que je tiens à remercier, pour son extrême obligeance). Fig. 2. -7 sections de Nectonema, au-dessous du cœur, entre les masses musculaires du céphalo-thorax de Leander (acide périodique-Schiff-vert lumière). FIG. 3. - 14 sections de Nectonema, de part et d'autre de la glande médio-intestinale (Fuchsine paraldehyde vert-lumière). FIG. 4. -8 sections de Nectonema, au voisinage de l'ovaire de l'hôte (acide périodique-Schiff-vert lumière). Fig. 5. - 10 sections, de Nectonema, après coloration par l'azan. FIG. 6. - Sections du Nectonema (coloration par l'hémalun picro-indigo-carmin) 


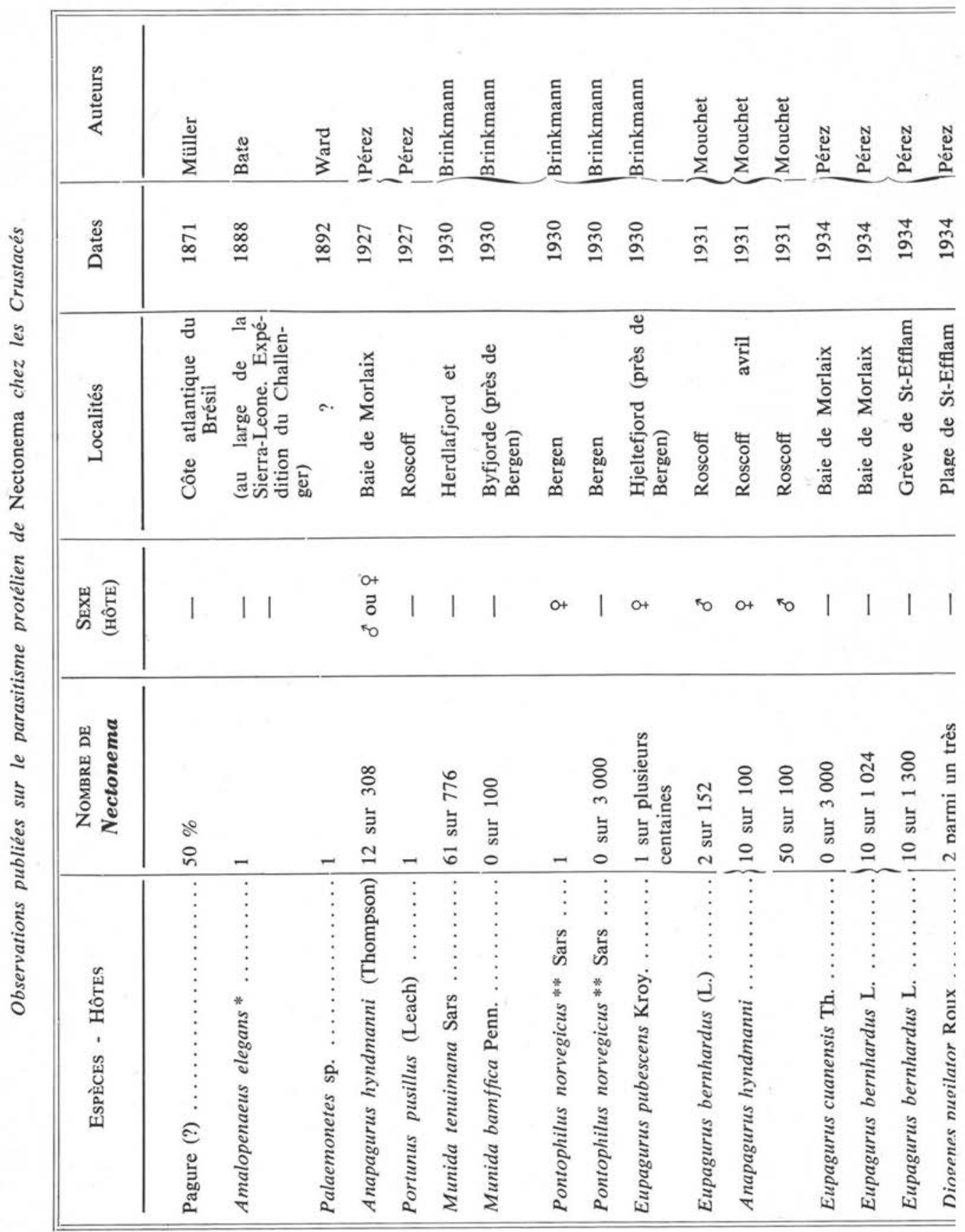


sance des appendices rompus expérimenta'ement, s'effectue chez les Crevettes parasitées comme chez les Crevettes témoins (Nouvel, 1934).

Le parasite est remarquab.ement bien supporté par les Crevettes : il est entièrement libre dans la cavité générale et ne provoque aucun réaction conjonctive (fig. 1 à 6). Au terme de son évolution il a emmagasiné une réserve énorme de métabolites variés, en particulier de polysaccharides (fig. 2 et 4). Cependant le Nectonema semble peu offensif et quand sa libération ne coïncide pas avec la mue de la Crevette il semble bien qu'il puisse périr sans que la Crevette en soit davantage affectée.

\section{Bibliographie}

BATE (S.), 1888. - Report scientific research. Challenger. Zool., 24, Crustacea Macrura.

BenNati-Mouchet (S.), 1934. - Castration parasitaire de l'Eupagurus prideauxi, par le Peltogaster curvatus. Trav. Stat. biol., Roscoff, $\mathrm{n}^{\circ} 12,11-9$.

Brinkmann (A.), 1930. - Ueber Nectonema munidae, n. sp., Bergens, Mus. Arbok, $\mathrm{n}^{\circ} 9$, 4-14.

Feyel (Th.), 1930. - Sur l'oogenèse du Nectonema agile Verr. C.R. Soc. Biol., 103, 681.

- 1930. - Sur la couche musculaire du Nectonema agile Verr. C.R. Soc. Biol., 103, 1174.

- 1936. - Recherches histologiques sur le Nectonema agile Verr. Etude de la forme parasite. Diplôme études supérieures Fac. Sci. Paris, pp. 36, 25 fig. et Arch. Anat. micr., 32, 197-234.

Gallien (L.), 1949. - Parasitisme protélien du genre Nectonema chez Leander squilla L et Portunus arcuatus Leach. Bull. Soc. Zool. Fr., 74, 179-84.

Huus (J.), 1931. - Ueber die Begattung bei Nectonema munidae Br. und über den Fund der Larve von dieser. Zool. Anz., 97, 33-7.

Mouchet (S.), 1931. - Spermatophores des Crustacés Décapodes Anomoures et Brachyoures et castration parasitaire chez quelques Pagures. Ann. Stat. Océanog Salambô, 6, pp. 203.

Müller (Fr.), 1871. - Bruchstüche zur Naturgeschichte der Bopyriden. Iena Z. Nat. wis., 6, 57.

Nouvel (H. et L.), 1934. - Sur deux Crevettes parasitées par un Nectonema. Evolution des parasites. Influence sur l'hôte. Bull. Soc. Zool. Fr., 59, 516-21.

- 1938. - Sur deux hôtes nouveaux de Nectonema. Bull. Mus., 10, 507-8.

Pérez (Ch.), 1927. - Sur le parasitsme protélien du Nectonema. C.R. Acad. Sci., 185, 226-7.

- 1927. - Sur le cycle évolutif du Nectonema. Xe Congrès internat. Zool., Budapest, p. 991.

- 1935. - Non spécificité du parasitisme du Nectonema agile Verrill. Bull. Soc. Zool. Fr., 59, 522-8.

- 1935. - Une ancienne observation relative au Nectonema. Bull. Soc. Zool. Fr., 60, 467-8.

WARD (H. B.), 1892. - On Nectonema agile Verrill. Bull. Mus., comp. Zool .Harvard Coll., 23, 135.

- 1893. - Preliminary communication on the host of Nectonoma agile Verrill. Proc. Amer. Acad. N.S., 19, 260.

(Laboratoire de Physiologie du C.N.R.Z., à Jouy-en-Josas et Laboratoire maritime de Dinard) 